\title{
New genome-wide methods bring more power to yeast as a model organism.
}

\section{John C Game}

Life Sciences Division, Lawrence Berkeley National Laboratory, 1 Cyclotron Road, Berkeley, CA 94720, USA.

email: jcgame@lbl.gov

Key-words: Yeast; Saccharomyces; DNA repair; Genomics; Microarrays; RAD genes; Model organisms.

A collection of 6,000 mutant yeast strains spanning nearly every gene offers new promise for identifying human genes involved in cellular responses to drugs, radiation and other treatments. 
Summary: Recent developments in genetics with Baker's Yeast, Saccharomyces cerevisiae, have provided quick new methods for identifying the genes that control cellular responses to treatment with a wide variety of agents, including chemicals and radiation. This greatly increases the power of yeast as a model system for studying human cellular responses to these agents, and for identifying human genes important in DNA repair and cancer.

The yeast Saccharomyces cerevisiae has long been used as a model organism for studying genetic and biochemical processes [1] (see also http://genomewww.stanford.edu/Saccharomyces/literature.html for online reviews). It is a unicellular microbial eukaryote combining a highly tractable molecular biology [2] with sexual reproduction and a very versatile genetic system. These features make it the species of choice for many studies of mitosis, meiosis, DNA repair and other aspects of biology shared by more complex life forms. Many yeast genes have homologues in human DNA, especially those involved in cell cycle control, meiosis and DNA repair [1]. Studying the yeast phenotypes of mutants in these genes leads to new knowledge of human genes important in cancer and other diseases.

\section{A complete mutant collection:}

All the DNA sequences comprising the genome of $S$. cerevisiae have been determined (see [3]). The genome sequence revealed that Baker's Yeast contains about 6,200 genes, including those currently defined only as open reading frames ("ORFs"), i.e., stretches of DNA whose sequence implies coding for a protein not yet identified. Each gene or ORF has been given a systematic name based on its physical position in the genome. The Saccharomyces genome database (http://genome-www.stanford.edu/Saccharomyces/) allows online searches for information concerning mutant phenotypes, literature references and other information using this name or any gene names associated with it.

Knowledge of these sequences has been put to good use. An international consortium has constructed a set of nearly six thousand yeast strains each lacking the function of a different gene or ORF [4]. The collection contains haploid and diploid strains separately disrupted for nearly every non-essential ORF, and diploid strains in which one copy has been disrupted for most genes that are essential. In each case, the gene has been replaced with an artificial DNA cassette integrated into the chromosome at the gene's location. The cassette contains a functional gene, KANMX4, conferring resistance to the antibiotic geneticin (G418). Wild-type yeast is sensitive to geneticin, hence cells containing the cassette (and thus lacking the original gene) can be identified by growth on medium containing the drug. In addition, each cassette contains short sequences on either side of the $K A N M X$ gene that are separate and unique for each gene or ORF that is deleted. These two sequences are known as the UPTAG and DOWNTAG sequences, since they uniquely tag each deletion and effectively function as a "barcode". Finally, the UPTAG and DOWNTAG sequences are themselves separately flanked by short sequences that are common to all the cassettes, permitting the barcode DNA to be amplified by PCR using these common sequences as primers [5]. 
The availability of this set of strains each containing a separate mutation, and together spanning nearly every yeast gene, provides a powerful new scientific resource [2]. Originally, in order to study the genetic control of a biological process, researchers treated wild-type yeast cells with mutagenic chemicals and identified survivors that demonstrated a chosen mutant phenotype. This needed painstaking follow-up work to identify which genes were mutated, and there was seldom any guarantee that a collection of mutants would identify all the genes that controlled the phenotype being studied.

Later, as reviewed by Ross-MacDonald [6], molecular methods were devised to create sets of mutants on a larger scale, for example by insertion of a lacZ reporter gene at random sites throughout the genome [7]. In this approach, the yeast gene involved is disrupted by a reporter gene that causes yeast colonies to turn blue whenever its own promoter is activated. Even these methods, however, have not generated a complete and catalogued set of yeast deletion mutants. With the new mutant set, it is easy to ask whether any given gene, when deleted, affects any chosen phenotype, rather than having to make new mutants each time and then work out which genes are affected. It is worth noting, however, that point mutations in some genes may confer phenotypes that are not seen in deletion mutants, and viable point-mutants may be useful for studying genes whose deletion alleles confer lethality. Hence, a role remains for new mutant isolation, despite the significance of the deletion set.

\section{Pooled mutants and microarrays:}

The area of DNA repair is a good example of the value of the deletions. Two groups have recently used the set of strains to identify new radiation-sensitive mutants in genes not previously known to affect this phenotype. First, Birrell et al. [8] used molecular methods to screen for ultraviolet radiation (UV) sensitivity simultaneously in 4,627 genedeleted diploid strains combined into a pool. These authors used UV to treat cultures of yeast containing all the strains mixed together. After irradiation, cells were allowed an 18 hour growth period to amplify the survivors and dilute out cells that were killed or severely growth-impaired by the treatment. DNA was then extracted from the cell population and all the barcode sequences were co-amplified by PCR, using the common flanking regions as primers. The objective was to amplify the barcode DNA while preserving the ratios in which each unique barcode was represented in the population. Finally, the amplified DNA was hybridized to high-density oligonucleotide microarray grids (DNA TAG3, Affymetrix, Santa Clara, CA.) containing sets of four DNA strands for each mutant in separate positions, each strand being complementary to one of the UPTAG or DOWNTAG barcode strands [5]. The authors reasoned that the relative intensity of hybridization signal at each grid position should reflect the proportion of the total cassette DNA contributed by the mutant whose unique barcode sequences were hybridized there. This in turn should reflect the relative cellular abundance of that mutant in the pool at the time of DNA extraction. Mutants that were killed more than average by the treatment should be under-represented, giving below-average signal in the positions containing their complementary barcode strands.

The authors averaged the signal intensity from the four barcode strands for each mutant and then determined each mutant's ratio of signal intensity with UV to that without UV. In most cases, this ratio did not vary greatly from gene to gene, but for some mutants, a ratio markedly below the average was seen. This implied a lower comparative cell 
survival for these mutants after UV treatment, leading to an under-representation of their DNA. In essence, the method represents a molecular way of doing UV survival assays on 4,627 mutants simultaneously.

The UV treatment results verified the methodology, since most genes previously known to be important in controlling UV-sensitivity (reviewed in [9]) were re-identified by the authors' screen. Some potential new UV-sensitive mutants were also identified, and in these cases UV survival assays involving colony formation were done to verify the initial observations. It is important to confirm sensitivity of new mutants in this way at several UV doses, and, in addition, to confirm that the sensitivity of the strain is conferred by the known deletion it contains. The survival assays showed that most, though not all, of the new strains initially identified by the hybridization signal intensity method were sensitive to UV killing. Birrell et al. [8] then transformed each new mutant with a wild-type copy of the gene, and observed that this step corrected the defect in the newly identified mutants. Hence the molecular method had identified some real new mutants and some false positives.

Another test for false positives involves crossing haploid strains containing the new mutation with wild-type, and determining if the studied phenotype is observed in only those progeny that inherit the deleted gene. If so, this confirms that the deletion is responsible for the phenotype. These crosses are also needed before more detailed study of the new mutants is undertaken, since independent changes including secondary mutations, aneuploidy, or spontaneous diploidisation can occur during yeast transformation. These may modify the mutants strain's phenotype even when this is conferred by the deletion itself.

The UV-sensitivity study was largely done to demonstrate the effectiveness of the method, since much is already known about UV-repair in Saccharomyces. The real promise of the genome-wide mutant collection and the screening method of Birrell et al. [8] lies in its application to other phenotypes. New pharmaceutical agents or toxic chemicals can be studied in the same way, and the genetic pathways determining their utilisation, breakdown, or responses to their effects may be identified more completely and with less work thanks to this approach.

\section{More specific mutant screens:}

The microarray approach was also used successfully by Ooi et al. [10] in a more specific assay that screened for mutants blocked in non-homologous end joining repair (NHEJ) [11]. This pathway is required in yeast for rejoining plasmids that are cut at sites with no homology to the yeast genome. These cannot be repaired by recombination, and are ineffective at transforming yeast unless they can be re-circularised by NHEJ. The authors used a marker on the plasmid to select for transformants in the whole pool of mutants and then determined the relative representation of each transformed mutant both with intact and with cut plasmid. Mutants relatively under-represented in the transformants from the cut plasmid compared to the intact one were largely those defective in NHEJ. A significant new gene, $N E J 1$, was identified and its phenotype was confirmed by other methods. 


\section{Directly testing the mutants:}

In another approach, Bennett et al. [12] used more conventional means to study radiationsensitivity in a subset of the new strain collection containing 3,670 homozygous diploid yeast deletion mutants. They individually spot-tested each mutant for sensitivity to a single dose of gamma rays. They made a preliminary identification of 107 new mutants showing gamma-ray sensitivity. Most of these mutants have not yet been confirmed as sensitive by rigorous survival assays. In addition, Bennett et al. did not confirm that the sensitivity of each presumptive mutant was conferred by the deleted gene, and in some cases their own data imply that it is not. They spot-tested the two haploid parents of 29 of the presumptive new mutant diploids. In 8 of these, at least one parent showed no gamma-ray sensitivity, despite containing the disrupted gene, and in another 3 mutants the sensitivity of one parent was much less than that of the other parent. The haploid parents of the remaining 78 mutants were not tested. Nevertheless, their study identified strains that can now be further assessed by well-established methods. A broadly similar approach was used earlier by Chan et al. [13] to screen for rapamycin-resistant mutants in a subset of the haploid deletion strains.

\section{Other ways to exploit the mutants:}

In a different use of the mutants, Tong et al. [14] devised a genetic strategy for quick identification of deletion mutants that are inviable when present in a double-mutant combination with any known mutant of interest. Such inviable doubles, known as synthetic lethals, can identify potential new genes that are involved in the same area of metabolism that is affected by the known gene. Using these newly found mutants themselves as the starting point for further synthetic lethal screens allows networks of synthetic lethality to be constructed in step-wise fashion, connecting ever-wider areas of metabolism [14]. This should eventually give enormous new information about pathways and about the third or so of yeast genes whose function still remains unknown.

\section{Looking ahead:}

The syergism between genome-wide molecular approaches and a mutant collection spanning an entire eukaryotic genome promises rapid progress towards a more global understanding of yeast gene functions in the near future. Many human genes code for specialised functions not present in yeast. However, the high homology between yeast and mammals for basic cell processes holds great promise for understanding human cell biology and disease genes as well as for better drug design [15].

Acknowledgement: The author thanks Martin Brown for comments on the manuscript. The author was supported by NIH Grant GM5997901 and DOE contract DE AC 76SF00098 while writing this article. 


\section{References:}

1 Resnick, M.A. and Cox, B.S. (2000) Yeast as an honorary mammal. Mutation Res. 451, 1-11

2 Kumar, A. and Snyder M. (2001) Emerging technologies in yeast genomics. Nat. Rev. Genet. 2, 302-312

3 Goffeau, A. et al. (1996) Life with 6,000 genes. Science 274, 563-567

4 Winzeler, E.A. et al. (1999) Functional characterization of the S. cerevisiae genome by gene deletion and parallel analysis. Science 285, 901-906

5 Shoemaker, D.D. et al. (1996) Quantitative phenotypic analysis of yeast deletion mutants using a highly parallel molecular bar-coding strategy. Nat. Genet. 14, 450456

6 Ross-Macdonald, P. (2000) Functional analysis of the yeast genome. Funct. Integr. Genomics 1, 99-113

7 Burns, N. et al. (1994) Large-scale analysis of gene-expression, protein localization, and gene disruption in Saccharomyces cerevisiae. Genes Dev. 8, 1087-1110

8 Birrell, G.W. et al. (2001) A genome-wide screen in Saccharomyces cerevisiae for genes affecting UV radiation sensitivity. Proc. Natl. Acad. Sci. U. S. A. 98, 1260812613

9 Game, J.C. (2000) The Saccharomyces repair genes at the end of the century. Mutation Res. 451, 277-293

10 Ooi, S.L. et al. (2001) A DNA microarray-based genetic screen for nonhomologous end-joining mutants in Saccharomyces cerevisiae. Science 294, 2552-2556

11 Paques, F. and Haber, J.E. (1999) Multiple pathways of recombination induced by double-strand breaks in Saccharomyces cerevisiae. Microbiol. Mol. Biol. Rev. 63, 349-404

12 Bennett, C.B. et al. (2001) Genes required for ionizing radiation resistance in yeast. Nat. Genet. 29, 426-434

13 Chan, T.F. et al. (2000) A chemical genomics approach toward understanding the global functions of the target of rapamycin protein (TOR). Proc. Natl. Acad. Sci. U.S.A. 97, 13227-13232

14 Tong, A.H. et al. (2001) Systematic genetic analysis with ordered arrays of yeast deletion mutants. Science 294, 2364-2368

15 Ma, D. (2001) Applications of yeast in drug discovery. Pro Drug Res. 57, 117-162 